\title{
Avaliação de risco e plasticidade comportamental limitada em girinos de Rhinella ornata (Anura, Bufonidae)
}

\author{
Renan N. Costa \& Fausto Nomura
}

Universidade Federal de Goiás (UFG), Departamento de Ecologia, Instituto de Ciências Biológicas, Laboratório de Herpetologia e Comportamento Animal, Campus Samambaia (Campus II). Av. Esperança, Caixa Postal 131, 74001-970, Goiânia, Goiás, Brasil. (renan.nunes.costa@gmail.com; fausto_nomura@yahoo.com.br)

\begin{abstract}
Assessment risk and limited behavioral plasticity in tadpoles of Rhinella ornata (Anura, Bufonidae). Anuran tadpoles are important elements of trophic networks in aquatic environments, being food resource for many types of predators. Thus, the tadpoles exhibit a great variety of defense mechanisms that may be morphological, behavioral and/or physiological. The unpalatability, produced by the accumulation of toxic substances in the skin, is a common mechanism in many frog lineages. However, some predators are not affected by these toxic substances, which may favor the development of alternative mechanisms of defense against predation. In this context, our objective was evaluate if the unpalatable tadpoles of Rhinella ornata (Spix, 1824) may present behavioral mechanisms of defense against predation in the presence of predators that are not affected by toxic substances on its skin. To test our hypothesis, we used two kinds of predators: an aquatic Heteroptera of the genus Belostoma and a dragonfly larva of the genus Aeshna. The tadpoles were located in aquariums with visual and chemical clues of predators (direct risk experiment), only chemical clues (indirect risk experiment) and by the complete absence of predator signals (control). In both cases, the swimming behavior was observed for 5 minutes. During the experiments there was no alteration in swimming behavior of tadpoles.
\end{abstract}

KEYWORDS. Predation, unpalatable, defensive behavior, invertebrate predators, chemical signs.

RESUMO. Os girinos de anuros são elementos importantes das redes tróficas de ambientes aquáticos, sendo recurso alimentar de diversos tipos de predadores. Desta maneira, os girinos apresentam uma grande variedade de mecanismos de defesa que podem ser morfológicos, comportamentais e/ ou fisiológicos. A impalatabilidade, produzida pelo acúmulo de substâncias tóxicas na pele, é um mecanismo comum em muitas linhagens de anfíbios. No entanto, alguns predadores não são afetados por estas substâncias tóxicas, o que pode favorecer o desenvolvimento de mecanismos alternativos de defesa contra predação. Neste contexto, nosso objetivo foi avaliar se girinos impalatáveis de Rhinella ornata (Spix, 1824), podem apresentar mecanismos comportamentais de defesa contra predação na presença de predadores que não são afetados pelas substâncias tóxicas em sua pele. Para testar nossa hipótese, utilizamos dois tipos de predadores aquáticos: um heteróptero aquático do gênero Belostoma e uma larva de libélula do gênero Aeshna. Os girinos foram colocados em aquários com pistas visuais e químicas dos predadores (experimento de risco direto), somente pistas químicas (experimento de risco indireto) e ausência completa de sinais de predadores (controle). Em ambos os casos, o comportamento de natação foi observado durante 5 minutos. Durante os experimentos não houve alteração no comportamento de natação dos girinos.

PALAVRAS-CHAVE. Predação, impalatabilidade, comportamento defensivo, predadores invertebrados, sinais químicos.

Conhecer as interações entre predadores e presas é um fator-chave para o entendimento de padrões e propriedades de comunidades naturais ( $\mathrm{S}_{\mathrm{IH}}$ et al., 1998; Werner \& PeAcor, 2003). Estudos experimentais tem evidenciado o papel dos predadores na regulação do tamanho populacional das presas ( $\mathrm{SiH}$ et al., 1985; LimA \& Dill, 1990), além da detecção e do reconhecimento de mecanismos antipredação, como a impalatabilidade e o comportamento críptico (NomuRA et al., 2011).

Os girinos de anfíbios anuros são bons modelos para estudos experimentais sobre predação, pois fazem parte da dieta de um grande número de vertebrados $(e$. $g$. peixes, aves, anfíbios) e invertebrados (e.g. baratas d'água, larvas de Odonata e aranhas) (Duellman \& Trueb, 1994). Geralmente os girinos apresentam alta plasticidade fenotípica, podendo alterar sua morfologia, fisiologia e/ou comportamento quando submetidos à pressão de predadores (McCollum \& VAN BuskiRK, 1996; Altig \& McDiarmid, 1999; Relyea, 2001; Moore et al., 2004). Dentre as respostas dos girinos aos predadores incluem a formação de agregados, abandono de microambiente, busca de refúgios, comportamento críptico, imobilidade ou redução da atividade de forrageio (ETERovick, 2000;
WeLLS, 2007). Neste caso, estas mudanças são geralmente induzidas por pistas indiretas, como o reconhecimento de substâncias químicas liberadas por predadores ou pela predação de coespecíficos (РеRотti et al., 2006), e por pistas diretas, como a presença física dos predadores e suas diferentes táticas de forrageio (WERNER et al., 1983; WARFE \& BARMUTA, 2004). Entretanto, a reação das presas pode ser mediada por componentes ambientais, como a estrutura do habitat ou pela presença de competidores, ou até pela forma como interagem com os predadores (Nomura et al., 2011, 2013). As diferentes respostas ao risco de predação são fundamentais para a coexistência de girinos e seus predadores (Hero et al., 2001)

Os girinos de algumas espécies apresentam coloração e comportamento críptico, o que diminui a chance de serem detectados em ambientes estruturados [e.g. Eupemphix nattereri (Steindachner, 1863)], enquanto outros apresentam coloração conspícua, o que é geralmente interpretado como uma condição de aposematismo [e.g. Rhinella schneideri (Werner, 1894), R. ornata (Spix, 1824), R. crucifer (Wied-Neuwied, 1821)], por destacar o girino no ambiente, criando uma advertência aos predadores sobre a presença de substâncias impalatáveis em sua pele 
(Eterovick, 2000; Wells, 2007; Nomura et al., 2011). No entanto, a eficiência dessas estratégias defensivas podem variar de acordo com o tipo de estratégia de forrageio (Hero et al., 2001; Nomura et al., 2011) e com o nível de sensibilidade às substâncias impalatáveis (Heyer et al., 1975; Hero et al., 2001) dos predadores. Por exemplo, a impalatabilidade é mais eficiente contra predadores que engolem a presa inteira, como peixes, ou que mastigam a presa antes de consumi-la, como larvas de Odonata (VAN BURSKIRK, 1988; Hero et al., 2001). Outros predadores que não engolem a presa ou a mastigam, como baratas-d'água do gênero Belostoma, não são afetados pela impalatabilidade (ToBler et al., 2007). As baratas-d'água injetam toxinas que paralisam a presa e liberam enzimas digestivas que causam necrose nos tecidos da vítima (Swart \& Felgenhauer, 2003). Algumas larvas de libélula (e.g., gênero Aeshna) também não são afetadas pela impalatabilidade, apesar de mastigarem suas presas (CROSSLAND \& ALFORD, 1998; Nomura et al., 2011), e são consideradas importantes predadoras de girinos (Hero et al., 2001).

Muitos estudos relatam que girinos de Rhinella alteram seu comportamento na presença de diferentes predadores, sendo que a resposta mais comum é a redução de sua atividade (Skelly \& Werner, 1990; Anholt et al., 1996; Perotti et al., 2006; StaV et al., 2007; JARA \& Perotti, 2009, 2010). No entanto, essas defesas induzidas são muito plásticas e podem ser influenciadas pelo estágio de desenvolvimento ontogenético do girino, diferentes níveis de palatabilidade, coexistência com predadores específicos, complexidade estrutural dos ambientes, entre outros (Relyea, 2003; Kopp et al., 2006; Richter-Boix et al., 2007; Jara \& Perotti, 2009). Por exemplo, a complexidade estrutural no interior dos corpos d'água pode aumentar o número de refúgios para os girinos e diminuir o risco de predação (TARR \& BABBitT, 2002; Kopp et al., 2006; SAHA et al., 2009), entretanto, diferentes substratos podem servir de poleiro e influenciar positivamente o sucesso de forrageio de predadores invertebrados, principalmente aqueles com estratégia do tipo senta-e-espera e guiados por pistas táteis e visuais, como as larvas de Odonata e belostomatídeos (Hemiptera) (PRITCHARD, 1965; Werner et al., 1983; Azevedo-Ramos et al., 1992; Warfe \& Barmuta, 2004; Tobler et al., 2007; Nomura et al., 2011).

Quando predadores de diferentes espécies ocorrem em um mesmo ambiente, a pressão e os efeitos sobre a população de presas podem variar de acordo com as interações entre os potenciais competidores (FINKE \& Denno, 2002). Interações entre predadores podem ter efeitos aditivos, sinérgicos ou antagônicos. Efeitos aditivos ocorrem quando a soma dos efeitos individuais dos predadores causa um maior impacto nas populações de presas (CHANG, 1966). Interações sinérgicas acontecem em situações onde um predador facilita o impacto do outro, podendo ser resultado de métodos de forrageio complementares (Losey \& DeNNo, 1998). Já as interações antagônicas podem ser mediadas por métodos de forrageio similares, onde o efeito de um predador se sobrepõe ao efeito do outro e vice-versa (Moran et al., 1996). Neste caso, há uma diminuição na captura de presas além da possível predação intraguilda (Polis \& HoLT, 1992).

Neste contexto, nosso objetivo foi avaliar se girinos de Rhinella ornata apresentam mecanismos comportamentais de defesa diante do risco de predação. Mais especificamente, testamos se na presença visual e química de predadores que não são afetados pelas substâncias impalatáveis, os girinos de $R$. ornata reduzem sua atividade natatória. Também avaliamos o efeito da presença de predadores competidores sobre as taxas de predação em girinos de $R$. ornata. Neste caso, esperamos que quando os indivíduos de Belostoma sp. e de Aeshna sp. ocorram juntos promovam um efeito aditivo sobre a população de presas, causando maior impacto em um menor tempo. Adicionalmente, testamos se apenas o sinal químico deixado pelo predador modifica o comportamento do girino. Neste caso, esperamos que o tempo de natação dos girinos seja reduzido com o sinal químico deixado pelo predador.

\section{MATERIAL E MÉTODOS}

Sistema de estudo. Os girinos de $R$. ornata foram coletados com um puçá de tela de arame em um corpo d'água lêntico de restinga próximo à praia $\left(23^{\circ} 21^{\prime} 31.42^{\prime \prime}\right.$; $44^{\circ} 21^{\prime 2} 23.66^{\prime \prime}$ ), totalizando 80 indivíduos. Os predadores invertebrados foram coletados em um corpo d'água em área de capoeira, totalizando 45 indivíduos de Belostoma sp. e 45 indivíduos de Aeshna sp. (23²1'38.49”'S; 4450’02.65”O). A coleta foi realizada no mês de julho de 2012 no núcleo Picinguaba do Parque Estadual da Serra do Mar, localizado no município de Ubatuba-SP e inserido no bioma Mata Atlântica.

Nos ambientes onde os predadores foram coletados retiramos amostras de folhiço e pedras para fornecer substrato adequado para o comportamento de captura e forrageio dos Belostoma sp. e Aeshna sp. A quantidade e disposição de substrato nos aquários foram mantidas uniformes entre as observações. Embora a complexidade estrutural do habitat possa reduzir o risco de predação (Finke \& Denno, 2002; Tarr \& Babbitt, 2002; Kopp et al., 2006; SAHA et al., 2009), esta atenuação só ocorre se a estrutura ambiental fornecer ambientes livre de inimigos (enemy-free spaces). Assim, a disposição do substrato foi organizada de modo a não criar refúgios para os girinos de $R$. ornata (e.g., espaço entre pedras ou embaixo de folhas) e fornecer aos predadores substrato adequado durante os experimentos, simulando as condições naturais do corpo d'água na expectativa de maximizar o seu potencial de forrageio.

Utilizamos girinos de $R$. ornata entre os estágios 35-39 (sensu GOSNER, 1960) [Comprimento total (mm): média \pm devio padrão (variação) $=21.19 \pm 1.85$ (18.83 - 23.97); $\mathrm{n}=11$ ]. Os predadores Belostoma sp. e Aeshna 
sp. foram selecionados visualmente para acomodar as seguintes condições: tamanho corporal semelhante e o comprimento total dos predadores de aproximadamente três vezes o comprimento total dos girinos. Os predadores coletados foram deixados sem alimento por 24 horas antes do início dos experimentos.

Em ambos os experimentos, utilizamos apenas um girino de $R$. ornata por aquário para isolar o comportamento de formação de agregados desta espécie. Assim, avaliamos apenas a taxa de atividade dos girinos, analisada ao nível de indivíduo. Em ambos os experimentos, cada unidade experimental foi observada durante 5 minutos e o tempo de deslocamento dos girinos mensurado com auxílio de um cronômetro digital. O tempo de deslocamento foi considerado apenas quando o girino se locomovia entre pontos distintos no aquário. Atividade de movimentação da cauda que não resultava em deslocamento não foi medido durante a observação. Os experimentos foram realizados no período diurno próximo ao ponto de coleta dos girinos. Pinças, aquários e peneiras foram individualizadas por tratamento, evitando misturar predadores e/ou compostos químicos liberados pelos mesmos. Antes de cada réplica, além de trocar o folhiço e a água, sempre substituímos os predadores e os girinos. Todos os indivíduos foram utilizados apenas uma vez por unidade experimental. Exemplares de girinos de $R$. ornata foram depositados na Coleção Herpetológica da Universidade Federal de Goiás - ZUFG 1508 e os predadores devolvidos aos ambientes onde foram capturados após os experimentos.

Experimento de risco direto. Para testar se os girinos modificam o seu comportamento de natação quando expostos ao risco de predação, realizamos um experimento criando microcosmos em quatro aquários plásticos redondos $(110 \mathrm{~cm}$ x $80 \mathrm{~cm})$, com $320 \mathrm{ml}$ de água limpa. Em cada microcosmo, os girinos foram submetidos à presença dos predadores nos seguintes tratamentos: controle $(\mathrm{Co})-$ sem a presença de predadores; Belostoma sp. (Bel) - dois indivíduos de Belostoma sp.; Aeshna sp. (Aes) - dois indivíduos de Aeshna sp.; e Misto (Mis) - um indivíduo de Belostoma sp. e um indivíduo de Aeshna sp. Desta forma, esperamos controlar o efeito da abundância de predadores entre os tratamentos. Por meio de sorteio, selecionamos a ordem das observações de cada tratamento. Os predadores foram adicionados ao mesmo tempo em todos os aquários e deixados para se aclimatar no novo ambiente durante um minuto. Os girinos foram adicionados aos aquários após o período de aclimatação dos predadores e as observações do comportamento do girino se iniciavam após 30 segundos. O tempo de aclimatação dos girinos foi menor, comparado ao tempo de aclimatação dos predadores, devido ao risco de predação (experimento letal). Nos aquários que ocorreram eventos de predação, a observação foi encerrada, o predador registrado e o tempo de deslocamento dos girinos ponderado em relação ao tempo total da observação do experimento. Realizamos 11 réplicas de cada tratamento, totalizando 44 aquários observados e aproximadamente $03 \mathrm{~h} 30 \mathrm{~min}$ de observação direta.

Experimento de risco indireto. No experimento de detecção do sinal químico, utilizamos o mesmo sistema detalhado no experimento anterior. Entretanto, foram adicionados apenas $80 \mathrm{ml}$ de água limpa e um girino de $R$. ornata em três aquários diferentes. Os girinos foram mantidos cinco minutos em cada aquário para aclimatação. Após esse período, o restante da água dos aquários foi completada com a água de dois aquários auxiliares onde 10 indivíduos de Belostoma sp. e 10 indivíduos de Aeshna sp. foram mantidos por 12 horas, com intuito de concentrar químicos liberados na água. Nos aquários, os girinos foram submetidos a três tratamentos: Controle (Contr) - adição de $240 \mathrm{ml}$ de água limpa; Belostoma sp. (Belos) - adição de $240 \mathrm{ml}$ de água dos aquários com indivíduos de Belostoma sp.; e Aeshna sp. (Aeshn) - adição de $240 \mathrm{ml}$ de água dos aquários com indivíduos de Aeshna sp. A água foi adicionada de forma gradual e cuidadosa ao aquário para reduzir o estresse hídrico. Por meio de sorteio, selecionamos a ordem das observações. Realizamos 10 réplicas de cada tratamento, totalizando 30 aquários observados e aproximadamente $02 \mathrm{~h} 30 \mathrm{~min}$ de observação direta.

Análise de dados. No experimento de risco direto realizamos uma ANOVA Fatorial para comparar o tempo de natação dos girinos em relação à presença e aos tipos de predadores no tratamento. Utilizamos como variável resposta a proporção do tempo de deslocamento, ponderada em relação ao tempo total de duração de cada experimento e cada predador como um fator (dois níveis, presença ou ausência). No experimento de risco indireto, realizamos uma ANOVA para comparar se apenas o sinal químico deixado pelo predador altera o comportamento de natação dos girinos entre os tratamentos (ZAR, 1999).

\section{RESULTADOS}

Não houve redução na atividade de natação dos girinos de R. ornata na presença de predadores Belostoma sp. $\left(\mathrm{F}_{(1,11)}=0,36 ; \mathrm{p}=0,54\right)$, na presença de larvas de Aeshna sp. $\left(\mathrm{F}_{(1,11)}=2,40 ; \mathrm{p}=0,12\right)$ ou na presença de ambos os predadores $\left(\mathrm{F}_{(1,11)}=1,65 ; \mathrm{p}=0,20\right)$ (Fig. 1). Desta maneira, os girinos de $R$. ornata apresentam atividade de deslocamento constante, independente do risco de predação ou do tipo de predador. Entretanto, apesar da ausência de resposta ao risco de predação, observamos nove eventos predatórios, quatro no tratamento Aes $(44,4 \%)$, com um evento resultando na morte do girino, e cinco no tratamento Mis. No tratamento Mis, todos os eventos de predação observados foram realizados por Belostoma sp. as quais capturaram tanto girinos (33,3\%) quanto larvas de Aeshna sp. (22,2\%).

No experimento de risco indireto, os girinos de $R$. ornata não apresentaram diferenças significativas no tempo de deslocamento entre os tratamentos aos quais foram expostos $\left(\mathrm{F}_{(2,10)}=0,06 ; \mathrm{p}=0,93\right)$ (Fig. 2). Desta maneira, girinos de $R$. ornata não distinguiram a presença ou tipo de predador em função das pistas químicas presentes na água. 


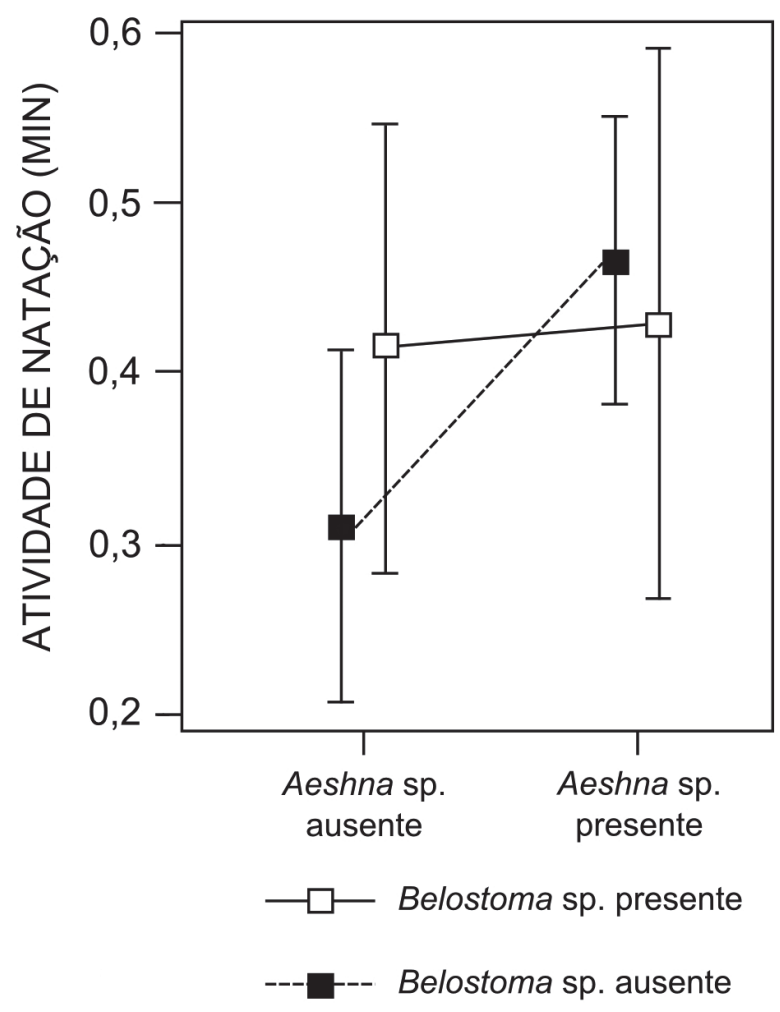

Fig. 1. Atividade de natação (min) de girinos de Rhinella ornata (Spix, 1824) em resposta aos estímulos visuais e químicos (experimento de risco direto) de dois tipos de predadores: Belostoma sp. (Hemiptera) e Aeshna sp. (Odonata). Os quadrados representam as médias e as barras verticais representam o intervalo de confiança $\pm 0,95$.

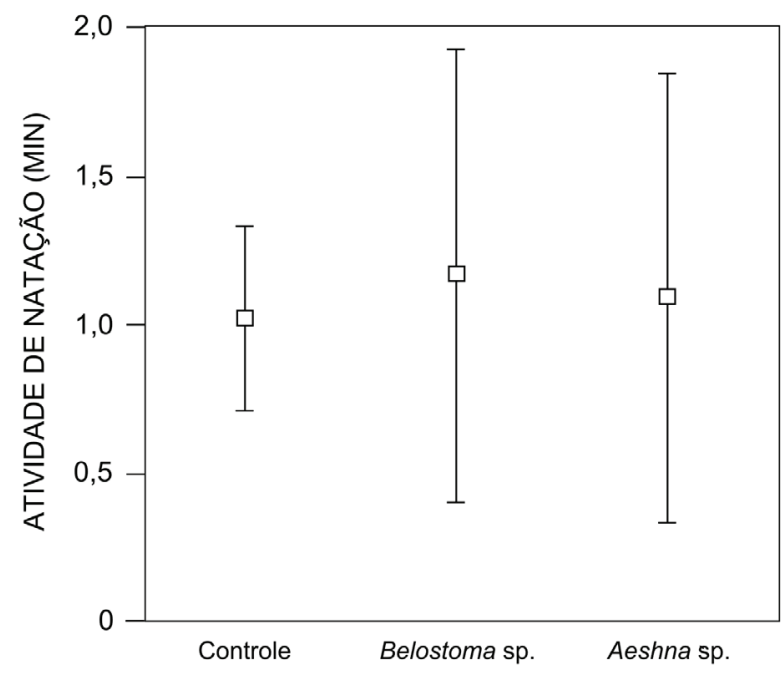

Fig. 2. Tempo de natação (min) de girinos de Rhinella ornata (Spix, 1824) em função das pistas químicas de diferentes predadores invertebrados (experimento de risco indireto). Os quadrados representam as médias e as barras verticais representam o intervalo de confiança $\pm 0,95$.

\section{DISCUSSÃO}

Plasticidade comportamental limitada. $\mathrm{Na}$ presença de predadores, os girinos podem apresentar diversos mecanismos antipredação (BRODIE JR. et al., 1991;
D’Heursel \& HadDAD, 1999), os quais são dependentes das normas de reação ou síndromes comportamentais das espécies ( $\mathrm{S}_{\mathrm{IH}}$ et al., 1985; KoOlHASs et al., 1999; JARA \& Perotti, 2010). Em geral, a resposta induzida mais comum é a redução da atividade natatória, o que reduz a visibilidade e a taxa de encontro do girino com potenciais predadores (AZEVEDo-RAMOs et al., 1992; RICHARDSON, 2001). O comportamento dos animais é considerado uma característica com grande plasticidade intraindividual, o que pode propiciar a maior aptidão possível dos indivíduos independentemente do contexto ecológico (veja uma revisão em SiH et al., 2003, 2004). Entretanto, existem algumas situações onde uma tendência comportamental é mantida entre diferentes contextos ecológicos, originando o que é reconhecido hoje como síndromes comportamentais (SiH et al., 2003, 2004). Neste caso, é necessário reconhecer que alguns comportamentos podem apresentar plasticidade fenotípica limitada, impedindo a exibição do ótimo comportamental em todos os contextos ecológicos possíveis (SIH et al., 2003, 2004). Este parece ser o caso do comportamento exploratório dos girinos de $R$. ornata. Em nossos experimentos, esperávamos que os girinos de $R$. ornata modulassem o tempo de deslocamento em função da presença e do tipo de predador, reduzindo sua atividade natatória como forma de compensar o risco à predação (WeRner \& ANHOLt, 1993). No entanto, não observamos nenhuma redução no tempo de deslocamento, independentemente do risco ou tipo de predador. Assim, os girinos de R. ornata devem apresentar uma tendência a maximizar o comportamento exploratório de forma independente do contexto ecológico, como já foi demonstrado para outros girinos de espécies de Rhinella (Nomura et al., 2011, 2013). Por exemplo, girinos de $R$. schneideri são sempre mais ativos que girinos de Eupemphix nattereri, independente da presença de competidores ou predadores, aumentando a aptidão durante interações competitivas, mas aumentando também o risco de predação (Nomura et al., 2011, 2013). Neste contexto, a flexibilidade fenotípica limitada pode levar a expressão de comportamentos aparentemente subótimos (SiH et al., 2003, 2004), o que favoreceria a evolução de características compensatórias para redução do custo da estratégia comportamental, como a impalatabilidade (Nomura et al., 2013). Provavelmente, em R. ornata, a impalatabilidade é um traço fixo que evoluiu para sustentar a maior atividade exploratória, compensando o risco à predação e maximizando o potencial competitivo da espécie. Este padrão de comportamento associado à plasticidade fenotípica limitada (ou seja, a manutenção da tendência de maior exibição do comportamento exploratório entre contextos ecológicos distintos) é também conhecido como padrão proativo ("proactive coping style") e pode ser vantajoso em ambientes de alta previsibilidade ambiental (KoolhaAs et al., 1999). A adoção do padrão proativo por $R$. ornata é independente da detecção de predadores, uma vez que os girinos não modularam a sua atividade 
na presença de pistas indiretas, embora a eficiência da estratégia de defesa, i.e. a impalatabilidade, seja sensível ao tipo de predador (Semlitsch \& Gavasso, 1992). A impalatabilidade não depende da detecção prévia de um predador para ser eficiente, mas sim, da sensibilidade do predador à substância impalatável e da capacidade de aprendizagem. Este tipo de estratégia pode favorecer a espécie em ambientes com baixa pressão de predadores ou em situações onde a impalatabilidade é eficiente como, por exemplo, em ambientes onde os peixes são os principais predadores (Hero et al., 2001; Nomura et al., 2011).

Múltiplos predadores. Diferentes tipos de predadores exercem diferentes pressões evolutivas sobre as populações de presas (BRODIE JR. et al., 1991), entretanto, os diferentes comportamentos predatórios podem reduzir a aptidão de uma espécie de predador na presença de outras espécies competidoras (FInKE \& DenNo, 2002). Geralmente, as larvas de Aeshna sp. são mais eficientes predando girinos que apresentam atividade natatória constante (Nomura et al., 2011). Em nosso experimento, observamos que a eficiência de predação de Aeshna sp. foi menor no tratamento Misto, o que pode sugerir uma tendência de interação antagônica entre Aeshna sp. e Belostoma sp. Um resultado semelhante foi encontrado por HUANG \& $\mathrm{SiH}$ (1991), que estudaram a interação entre peixes (Lepomis sp.) e salamandras (Ambystoma sp.) se alimentando de larvas de isópodos (Lirceus sp.). As salamandras sozinhas se alimentam mais de isópodos do que os peixes, porém, quando os predadores estão juntos, a situação se inverte. Com a presença dos peixes, as salamandras buscam refúgios que são similares aos usados pelos isópodos, os quais ficam mais expostos ao risco de predação, aumentando o sucesso dos peixes. Estas interações antagônicas entre predadores podem reduzir o risco de predação das presas (Siн et al., 1998).

Em nossos experimentos, buscamos replicar as condições nas quais os predadores forrageiam e capturam suas presas, adicionando pedras e folhiço ao aquário. Geralmente, a complexidade estrutural do habitat somado ao efeito de múltiplos predadores pode reduzir o risco de predação de girinos (FInKe \& Denno, 2002; TARR \& Bаввітt, 2002; Kopp et al., 2006; SAHA et al., 2009). Entretanto, esta atenuação só ocorre se a estrutura ambiental fornecer ambientes livre de inimigos (enemy-free spaces). Em outras condições, por exemplo, quando diferentes tipos de predadores co-ocorrem, a estrutura física do ambiente pode ser importante para definir os papéis ecológicos das espécies envolvidas na rede de interações (Pritchard, 1965; Werner et al. 1983; Azevedo-Ramos et al, 1992; WARFE \& BARMUTA, 2004; Tobler et al., 2007; Nomura et $a l ., 2011)$. Isso pode fazer com que uma das espécies de predadores assuma o papel de predador de topo, reduzindo o número de predadores no ambiente e atenuando a força da predação sobre os girinos. Uma vez detectado este efeito antagônico da predação intraguilda (PoLIS \& HoLT, 1992), provavelmente os indivíduos de Belostoma sp. reduzem o impacto da predação causada por larvas de Aeshna sp. sobre as populações de girinos. Entretanto, esta hipótese necessita de comprovação em estudos futuros.

Conclusão. O comportamento de natação dos girinos de $R$. ornata não foi afetado pela presença ou pistas indiretas da presença dos predadores. Aparentemente, $R$. ornata apresenta uma plasticidade comportamental limitada nas suas respostas antipredatórias, o que pode ser vantajoso em ambientes de alta previsibilidade. Belostoma sp. pode afetar positivamente as populações de girinos, uma vez que pode reduzir as populações de Aeshna sp., no entanto, esta hipótese necessita ser comprovada por estudos futuros.

Agradecimentos. Agradecemos ao Prof. Dr. Gustavo Q. Romero e Prof. Dr. Martin Pareja pelo auxílio e discussões sobre o trabalho. Ao Prof. Dr. Natan M. Maciel pela revisão do inglês. Agradecemos também ao curso de Pós-graduação em Ecologia da Unicamp, ao curso de Pós-graduação em Ecologia e Evolução da UFG e à equipe do núcleo Picinguaba do Parque Estadual da Serra do Mar pelo apoio e oportunidade oferecida.

\section{REFERÊNCIAS BIBLIOGRÁFICAS}

Azevedo-Ramos, C.; Van Sluys, M.; Hero, J. M \& Magnusson, W. E. 1992. Influence of tadpole movement on predation by odonata naiads. Journal of Herpetology 26:335-338.

Anholt, B. R.; Skelly, D. K. \& Werner, E. E. 1996. Factors modifying antipredator behavior in larval toads. Herpetologica 52(3):301-313.

Altig, R. \& McDiarmid, R. W. 1999. Tadpoles. The biology of anuran larvae. Chicago, London, University of Chicago Press. 444p.

Brodie Jr., E. D.; Formanowicz JR., D. R. \& Brodie III, E. D. 1991. Predator avoidance and antipredator mechanisms: distinct pathways to survival. Ethology, Ecology and Evolution 3:73-77.

Chang, G. C. 1996. Comparison of single versus multiple species of generalist predators for biological control. Environmental Entomology 25:207-212.

Crossland, M. R. \& Alford, R. A. 1998. Evaluation of the toxicity of eggs, hatchlings and tadpoles of the introduced toad Bufo marinus (Anura: Bufonidae) to native Australian aquatic predators. Austral Ecology 23:129-137.

D'Heursel, A. \& Haddad, C. F. B. 1999. Unpalatability of Hyla semilineata tadpoles (Anura) to captive and free-ranging vertebrate predators. Ethology, Ecology Evolution 11:339-348.

Duellman, W. E. \& Trueb, L. 1994. Biology of amphibians. Baltimore, London, The Johns Hopkins University Press. 696p.

Eterovick, P. C. 2000. Effects of aggregation on feeding of Bufo crucifer (Anura, Bufonidae). Copeia 1:210-215.

Finke, D. L. \& Denno, R. F. 2002. Intraguild predation diminished in complex-structured vegetation: implications for prey suppression. Ecology 83:643-652.

Gosner, K. L. 1960. A simplified table for staging anuran ambryos and larvae with notes on identification. Herpetologica 16:183-190.

Hero, J. M.; Magnusson, W. E.; Rocha, C. F. D. \& Catterall, C. P. 2001. Antipredator defenses influence the distribution of amphibian prey species in the central Amazon rain forest. Biotropica 33:131-141.

Heyer, W. R.; McDiarmid, R. W. \& Weigmann, D. L. 1975. Tadpoles, predation and pond habits in the tropics. Biotropica 7:100-111.

HuAng, C. \& SiH, A. 1991. Experimental studies on direct and indirect interactions in a three trophic-level stream system. Oecologia 85:530-536.

Jara, F. G. \& Perotti, M. G. 2009. Toad tadpole responses to predator risk: ontogenetic change between constitutive and inducible defenses. Journal of Herpetology 43:82-88.

2010. Risk of predation and behavioural response in three anuran species: influence of tadpole size and predator type. Hydrobiologia 644:313-324.

Koolhaas, J. M.; Korte, S. M.; De Boer, S. F.; Van Der Vegt, B. J.; Van Reenen, C. G.; Hopster, H.; De Jong, I. C.; Ruis, M. A. W. \& Blokhuis, H. J. 1999. Coping styles in animals: current status in 
behavior and stress-physiology. Neuroscience and Biobehavioral Reviews 23:925-935.

Koop, K.; Wachlevski, M. \& Eterovick, P. C. 2006. Environmental complexity reduces tadpole predation by water bugs. Canadian Journal of Zoology 84:136-140.

Lima, S. L. \& Dill, L. M. 1990. Behavioral decisions made under the risk of predation: a review and prospectus. Canadian Journal of Zoology 68:619-640.

Losey, J. E. \& DenNo, R. F. 1998. Positive predator-predator interactions: enhanced predation rates and synergistic suppression of aphid populations. Ecology 79:2143-2152.

McCollum, S. A. \& VAn Buskirk, J. 1996. Costs and benefits of a predator-induced polyphenism in the gray treefrog Hyla chrysoscelis. Evolution 50(2):583-593.

Moore, R. D.; Griffiths, R. A.; O’Brien, C. M.; Murphy, A. \& Jay, D. 2004. Induced defences in an endangered amphibian in response to an introduced snake predator. Oecologia 141:139-147.

Moran, M. D.; Rooney, T. P. \& HuRd, L. E. 1996. Top-down cascade from a bitrophic predator in an old-field community. Ecology 77:22192227.

Nomura, F.; De Marco, P.; Carvalho, A. F. A. \& Rossa-Feres, D. C. 2013. Does background colouration affect the behaviour of tadpoles? An experimental approach with an odonate predator. Ethology, Ecology and Evolution 25(2): 185-189.

Nomura, F.; Prado, V. H. M.; Silva, F. R.; Borges, R. E.; Dias, N. Y. N. $\&$ Rossa-Feres, D. C. 2011. Are you experienced? Predator type and predator experience trade-offs in relation to tadpole mortality rates. Journal of Zoology 288:144-150.

Perotti, M. G.; Fitzgerald, L. A.; Moreno, L. \& Pueta, M. 2006. Behavioral responses of Bufo arenarum tadpoles to odonate naiad predation. Herpetological Conservation and Biology 1:117-120.

Polis, G. A. \& Holt, R. D. 1992. Intraguild predation: the dynamics of complex trophic interactions. Trends in Ecology and Evolution 7:151-154.

Pritchard, G. 1965. Prey capture by dragonfly larvae (Odonata: Anisoptera). Canadian Journal of Zoology 43:271-289.

RelyeA, R. A. 2001. Morphological and behavioral plasticity of larval anurans in response to different predators. Ecology 82(2):523-540. . 2003. Predators come and predators go: the reversibility of predator-induced traits. Ecology 84(7):1840-1848.

Richardson, J. L. 2001. A comparative study of activity levels in larval anurans and response to the presence of different predators. Behavioral Ecology 12:51-58.

Richter-Boix, A.; Llorente, G. A. \& Montori, A. 2007. A comparative study of predator-induced phenotype in tadpoles across a pond permanency gradient. Hydrobiologia 583:43-56.

SAHA, N.; AditYA, G. \& SAHA, G. K. 2009. Habitat complexity reduces prey vulnerability: An experimental analysis using aquatic insect predators and immature dipteran prey. Journal of Asian-Pacific Entomology 12:233-239.
Semlitsch, R. D. \& Gavasso, S. 1992. Behavioral responses of Bufo bufo and Bufo calamita to chemical cues of vertebrate and invertebrate predators. Ethology, Ecology and Evolution 4:165-173.

Sin, A.; Bell, A. \& Johnson, J. C. 2004. Behavioral syndromes: an ecological and evolutionary overview. Trends in Ecology and Evolution 19(7):372-378.

SiH, A.; Englund, G. \& Wooster, D. 1998. Emergent impacts of multiple predators and prey. Trends Ecology and Evolution 13:350-355.

Sin, A.; Kats, L. B. \& Maurer, E. F. 2003. Behavioural correlations across situations and the evolution of antipredator behavior in a sunfish-salamander system. Animal Behaviour 65:29-44.

Sih, A.; Crowley, P.; McPeek, M.; Petranka, J. \& Strohmeier, K. 1985. Predation, competition, and prey communities: A review of field experiment. Annual Review of Ecology and Systematics 16:269311.

Skelly, D. K. \& Werner, E. E. 1990. Behavioral and life-historical responses of larval American toads to an odonate predator. Ecology 71(6):2313-2322.

Stav, G.; Kotler, B. P. \& Blaustein, L. 2007. Direct and indirect effects of dragonfly (Anax imperator) nymphs on green toad (Bufo viridis) tadpoles. Hydrobiologia 579:85-93.

Swart, C. \& Felgenhauer, B. 2003. Structure and function of the mouth-parts and salivary gland complex of the giant waterbug, Belostoma lutarium (Stal) (Hemiptera: Belostomatidae). Annals of the Entomological Society of America 96:870-882.

TARR, T. L. \& BABBITT, K. J. 2002. Effects of habitat complexity and predator identity on predation of Rana clamitans larvae. AmphibiaReptilia 23:13-20.

Tobler, M.; Schlupp, I. \& Plath, M. 2007. Predation of a cave fish (Poecilia mexicana, Poeciliidae) by a giant water-bug (Belostoma, Belostomatidae) in a Mexican sulphur cave. Ecological Entomology 32:492-495.

VAN BUSKIRK, J. 1988. Interactive effects of dragonfly predation in experimental pond communities. Ecology 69:857-867.

WARFE, D. M. \& BARMUTA, L. A. 2004. Habitat structural complexity mediates the foraging success of multiple predator species. Oecologia 141:171-178.

WelLs, K. D. 2007. The Ecology and Behavior of Amphibians. Chicago, London, The University of Chicago Press. 1148p.

Werner, E. E. \& Anholt, B. R. 1993. Ecological consequences of the trade-offs between growth and mortality rates mediated by foraging activity. The American Naturalist 142:242-272.

Werner, E. E.; Gilliam, J. F.; Hall, D. J. \& Mittelbach, G. G. 1983. An Experimental Test of the Effects of Predation Risk on Habitat Use in Fish. Ecology 64:1540-1548.

Werner, E. E. \& Peacor, S. D. 2003. A Review of Trait-Mediated Indirect Interactions in Ecological Communities. Ecology 84(5):1083-1100.

ZAR, J. 1999. Biostatistical analysis. Upper Saddle River, Prentice Hall. 944 p. 